\title{
Neuroscience of Exercise: Perspectives for Exercise Adherence
}

\author{
Bruno Ribeiro Ramalho Oliveira* \\ *Psychiatry and Mental Health Postgraduate Program, Psychiatry Institute, Federal University of Rio \\ de Janeiro, Rio de Janeiro, Brazil
}

It is well established that physical inactivity may increase the risk for the development of chronic diseases [1]. Also, physical inactivity is related to high costs with public health [2] therefore, regular exercise seems to be critical for individual and public health. In this context, it has been hypothesized that the affective response (pleasure and displeasure) may be a determinant of behavior [3], based on this premise, several original studies were performed during the last years as an attempt to establish a relationship between aerobic exercise and affective responses [4-7]. In general, the scientific literature demonstrates that aerobic exercise performed at high intensities is related to negative affective responses [8,9]. Moreover, a review published in 2015 showed that the affective response during aerobic exercise at a moderate intensity may predict future physical activity behavior [10]. However, despite the advancement of scientific literature in understanding the relationship between exercise and affective responses, the cerebral mechanisms that underlie this relationship are not well understood.

Different theories are used to explain how the brain processes the affective responses. Davidson, proposed that the asymmetric electrical activity of right and left prefrontal cortex (PFC) may explain the pattern of affective response [11]. Specifically, Davidson proposed that the higher activation of left PFC is linked to positive affective response by inhibiting the amygdala whereas the higher activation of right PFC is linked to negative affective response [12]. However, it should be considered that the amygdala and the PFC are not the only structures involved in the affect modulation. The hippocampus, insula and anterior cingulate cortex are also involved in the affect modulation [12]. For example, ventral hippocampus is linked to the reward circuitry and stress response (controlling the hypothalamic-pituitary-adrenal axis) [13] which may influence the affective responses [14].

In addition to the electrical and anatomical factors, metabolism is also an important factor to understanding the pattern of affective responses. Several substances such as cortisol [14], epinephrine and norepinephrine [14] and dopamine [15] may influence the affective responses. Another hypothesis used to explain the relationship between exercise and pleasure is the endocannabinoid hypothesis [16] which is based on the premise that an interaction between endocannabinoids and dopamine may influence the reward system increasing the sense of well being. Although research in this area has advanced, one of the main challenges is to understand how the brain interprets sensory inputs to generate an effective response.

In summary, the advancement of technology brings new perspectives for neuroscience of exercise. Different instruments may be used to assess cerebral responses to exercise such as electroencephalography, near-infrared spectroscopy or magnetic resonance. However, due to the problems associated with the use of these instruments during the exercise sessions, most of the studies performed to date used these instruments pre or post the exercise session. Therefore, future studies should focus on the use of these instruments during the exercise session. Moreover, considering that exercise may chronically influence cerebral circuitry, these mechanisms should be clarified in the context of acute and chronic responses to exercise.

\section{REFERENCES}

[1] Blair, S.N., Physical inactivity: the biggest public health problem of the 21st century. Br J Sports Med, 2009. 43(1): p. 1-2.

[2] Schenck, A., et al., America's health rankings: a call to action for individuals \& their communities. 2014 ed. 2014, Minnetonka: United Health Foundation. 146.

[3] Cabanac, M., Physiological role of pleasure. Science, 1971. 173(4002): p. 1103-7. 
[4] Ekkekakis, P., E.E. Hall, and S.J. Petruzzello, The relationship between exercise intensity and affective responses demystified: to crack the 40-year-old nut, replace the 40-year-old nutcracker! Ann Behav Med, 2008. 35(2): p. 136-49.

[5] Martinez, N., et al., Affective and Enjoyment Responses to High-Intensity Interval Training in Overweight-to-Obese and Insufficiently Active Adults. J Sport Exerc Psychol, 2015. 37(2): p. 138-49.

[6] Oliveira, B.R., et al., Continuous and high-intensity interval training: which promotes higher pleasure? PLoS One, 2013. 8(11): p. e79965.

[7] Parfitt, G., E.A. Rose, and W.M. Burgess, The psychological and physiological responses of sedentary individuals to prescribed and preferred intensity exercise. Br J Health Psychol, 2006. 11(Pt 1): p. 39-53.

[8] Ekkekakis, P., G. Parfitt, and S.J. Petruzzello, The pleasure and displeasure people feel when they exercise at different intensities: decennial update and progress towards a tripartite rationale for exercise intensity prescription. Sports Med, 2011. 41(8): p. 641-71.

[9] Oliveira, B.R., A.C. Deslandes, and T.M. Santos, Differences in exercise intensity seems to influence the affective responses in self-selected and imposed exercise: a meta-analysis. Front Psychol, 2015. 6: p. 1105.

[10] Rhodes, R.E. and A. Kates, Can the Affective Response to Exercise Predict Future Motives and Physical Activity Behavior? A Systematic Review of Published Evidence. Ann Behav Med, 2015. 49(5): p. 715-31.

[11] Davidson, R.J., What does the prefrontal cortex "do" in affect: perspectives on frontal EEG asymmetry research. Biol Psychol, 2004. 67(1-2): p. 219-33.

[12] Davidson, R.J., Affective neuroscience and psychophysiology: toward a synthesis. Psychophysiology, 2003. 40(5): p. 655-65.

[13] Sahay, A. and R. Hen, Adult hippocampal neurogenesis in depression. Nat Neurosci, 2007. 10(9): p. 1110-5.

[14] Ekkekakis, P. and S.J. Petruzzello, Analysis of the affect measurement conundrum in exercise psychology: IV. A conceptual case for the affect circumplex. Psychol Sport Exerc, 2002. 3(1): p. 35-63.

[15] Matta Mello Portugal, E., et al., Neuroscience of exercise: from neurobiology mechanisms to mental health. Neuropsychobiology, 2013. 68(1): p. 1-14.

[16] Dietrich, A. and W.F. McDaniel, Endocannabinoids and exercise. Br J Sports Med, 2003. 38(5): p. 536-41. 